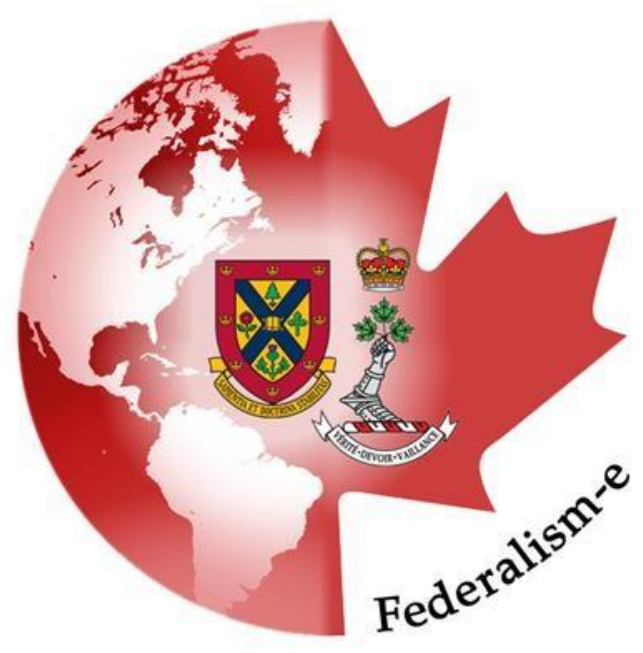

\title{
L'inclusion du droit à la sécession dans la constitution
}

Alex Miller-Pelletier, Université Laval

\section{Introduction}

La question de la sécession est une question qui touche beaucoup d'États, mais en particulier les pays qui sont composés d'États fédérés ou qui connaissent une certaine forme de dévolution envers des régions spécifiques de leur territoire. Dans les Constitutions des États fédéraux, plusieurs dispositions sont prévues, notamment les compétences respectives des paliers de pouvoir ainsi que les compétences résiduaires. Les dispositions concernant le droit à la sécession sont très rarement incluses dans les Constitutions fédérales. En ce moment, seules les Constitutions des fédérations de l'Éthiopie et de SaintKitts-et-Nevis mentionnent explicitement un droit à la sécession (Kreptul, 2003, p. 79). Il apparaît peut-être contraire à l'idée même de la fédération que de prévoir comment se séparer alors que les membres sont en train de s'unir. Pourtant, n'est-il pas légitime de croire que les fédérations ont un devoir d'inclure ses dispositions si l'on considère que les États fédérés ne sont pas inférieurs à l'État central et pourraient changer d'idée à tout moment? C'est ici qu'entrent en compte quelques distinctions qui pourraient influencer 
cette question, notamment dans le cas des États où le régime politique est un État unitaire plutôt qu'une fédération ou lorsqu'il est mentionné dans la Constitution que l'État central a un pouvoir sur les États fédérés. Il m'apparaît d'autant plus essentiel d'essayer de trouver une réponse à cette question, lorsqu'on sait que certains auteurs questionnent " la moralité et la légalité d'une sécession »1 (Kreptul, 2003, p. 39). En effet, ces auteurs ne voient pas en quoi une sécession est légitime, et encore moins l'inclusion d'un droit à la sécession dans une Constitution. Pourtant, ce droit et ses modalités devraient, à mon avis, être inclus dans les Constitutions des fédérations pour respecter l'essence du fédéralisme. Je reviendrai plus en détails sur les arguments avec lesquels je suis en accord par rapport à cette question. Je défendrai la position qui veut que les États devraient mettre un droit à la sécession dans leur Constitution.

\section{Justification et définition des termes}

Il importe de définir les termes présents dans la question de recherche de ce travail et de justifier le choix des termes afin d'obtenir des arguments précis. D'abord, la définition du terme sécession contient parfois un angle biaisé qui rappelle les idées soutenues par ceux qui ont écrit la définition en question. Par exemple, Lea Brilmayer évoque un « reniement fait par un groupe de personnes de leur obligation à respecter les lois de l'État » dans sa définition de la sécession (Brilmayer citée par Kreptul, 2003, p. 40). L'utilisation du mot " reniement" transmet tout de suite une connotation négative. Une définition neutre de ce qu'est le processus de la sécession, et celle qui sera utilisée dans ce texte, est celle de Julie Dahlitz: «lorsqu'une proportion importante de la population d'un territoire donné, faisant partie d'un État, exprime le souhait, par mot ou par acte, de devenir un État souverain ou de joindre un autre État » (Dahlitz, 2003, p. 6).

J'ai aussi fait le choix de concentrer cette recherche sur l'inclusion du droit à la sécession dans la Constitution bien que certains, dont Brilmayer, croient que « [l]e droit à la sécession est un enjeu de droit international» (Brilmayer, 1991, p. 177). Elle a en partie raison, puisqu'une reconnaissance internationale est nécessaire afin qu'un nouvel État soit considéré comme tel, mais, à mes yeux, il s'agit surtout d'un enjeu national. Il faudrait

\footnotetext{
${ }^{1}$ Toutes les citations présentées dans ce texte sont des traductions libres de l'anglais excepté les citations de Éthier (2004) et Hoffman (1996).
} 
d'abord que le droit à la sécession soit reconnu dans un État, puis que la communauté internationale le reconnaisse.

Le choix de terme le plus important de la question de recherche concerne le terme fédération. Une fédération se définit ainsi : "mode d'organisation politique qui unit des États distincts [...] dans un système politique global de façon à ce que chaque État puisse conserver son intégrité politique fondamentale»(Encyclopaedia Britannica, 2016). J'ai décidé de me concentrer sur les fédérations puisque ce sont des États qui sont constituées d'entités déterminées. La raison principale de ce choix est que les entités fédérées possèdent déjà trois des cinq caractéristiques propres aux États souverains, ce qui fait qu'il n’y a pas de discussions à avoir quant à la faisabilité d'instaurer ces caractéristiques. Les cinq caractéristiques du droit international que doit posséder un État pour être reconnu en tant que tel sont « un espace territorial, une population, un système de gouvernement, une personnalité juridique internationale [et] la souveraineté » (Éthier, 2004, p. 77). Dans le cas des fédérations, les entités fédérées possèdent déjà les trois premiers éléments et les deux derniers sont acquis lors de la reconnaissance de la sécession par la communauté internationale. Le fait qu'elles détiennent déjà trois des cinq éléments essentiels permet que mon argumentaire se concentre sur les raisons d'inclure un droit à la sécession dans la Constitution et non sur la faisabilité de la création d'un nouvel État indépendant. Par ailleurs, Altman et Wellman mentionnent que «les sécessionnistes peuvent se qualifier pour obtenir le droit à la sécession tant que leur communauté est à la fois capable et désireuse de remplir les fonctions politiques requises " (Altman et Wellman, 2009, p. 1). Le même raisonnement est alors utilisé pour le territoire: une entité fédérée a déjà pu démontrer ses capacités à exercer les responsabilités qui incombent à un gouvernement. En effet, l'argument voulant que la séparation du territoire, de la population et des pouvoirs entre l'État unitaire et l'État en devenir soit bien trop compliquée n'a pas raison d'être lorsque la recherche ne concerne que les fédérations. Dépendamment des arguments concernant le droit à la sécession, ils peuvent cependant s'appliquer parfois aux revendications sécessionnistes présentes dans les États unitaires. Ensuite, la fédération a l'avantage d'être constituée d'entités déjà formées et dont le territoire correspond fort probablement aux délimitations d'une population qui se rassemble autour de certains traits culturels ou linguistiques, comme c'est le cas avec le Québec par exemple. Enfin, le choix de 
ne parler que des fédérations s'explique aussi par un argument souvent formulé à l'encontre du droit à la sécession. Brilmayer le souligne, il existe, selon elle, une " pente très glissante dans la formulation d'un droit à la sécession qui n'ouvrirait pas la porte à une anarchie totale» (Brilmayer, 1991, p. 183). En se concentrant uniquement sur les fédérations, le droit à la sécession se limite aux entités fédérées et il n'y a donc plus lieu de discuter un désir de sécession pour une ville par exemple. Ce débat pourrait toutefois faire l'objet d'une autre recherche, mais je ne m’attarderai pas là-dessus dans celle-ci.

\section{Arguments contre}

Bien que je défende la présence d'un droit à la sécession dans la Constitution d'un État, je présente aussi brièvement les arguments qui sont contre afin de présenter une vue d'ensemble de l'enjeu. J'ai répertorié trois arguments principaux qui sont en défaveur de l'intégration d'un droit à la sécession dans la Constitution.

Premièrement, certains soutiennent l'idée qu'un droit à la sécession mènerait à "l'instabilité politique et au chaos" (Kreptul, 2003, p. 50). Ces auteurs croient que, quotidiennement, les politiciens feraient face à des débats concernant le droit à la sécession et que celui-ci serait discuté plutôt que les "vrais" enjeux politiques (Sunstein, 1991, p. 634). La question du droit à la sécession finirait par être mêlée directement aux questions politiques du moment (Sunstein, 1991, p. 634). Dans un même ordre d'idées, Sunstein déclare aussi que le droit à la sécession dans la Constitution risque de changer la conduite des acteurs politiques (1991, p. 634). Il mentionne à plusieurs reprises l'éventualité que les politiciens adoptent des "comportements stratégiques" (Sunstein, 1991, p. 634). Il donne l'exemple d'une entité fédérée qui aurait beaucoup de pouvoir économique et qui s'en servirait pour faire pencher la balance de son côté dans la discussion de tout enjeu politique, sous menace de recourir au droit à la sécession de la Constitution (Sunstein, 1991, p. 648-49). En fait, Sunstein croit que l'inclusion d'un droit à la sécession dans la Constitution menace la "planification sur le long-terme" des politiques gouvernementales (1991, p. 650). Selon leurs interprétations, le droit à la sécession monopoliserait toutes les discussions politiques et les politiciens se chargeraient principalement de calmer les menaces sécessionnistes et non plus à prévoir l'avenir de l'État. Je ne suis pas en accord 
avec cet argument et ma réponse à celui-ci se trouve dans le premier argument présenté en faveur de l'inclusion d'un droit à la sécession dans la Constitution.

Deuxièmement, la question de la justice est très importante dans le débat du droit à la sécession, et pas seulement lorsqu'il est question de l'inclure dans une Constitution. Selon le philosophe Rawls, un "État démocratique constitutionnel est nécessairement un État “juste” parce que c'est le seul type d'organisation politique qui peut assurer et protéger les droits humains de base et des droits politiques égaux pour tous les citoyens " (Rawls cité par Kreptul, 2003, p. 45). C'est en suivant cette perspective que le droit à la sécession est présenté comme n'ayant pas lieu d'être; il n'est pas nécessaire de se séparer si la justice est déjà présente. Cela présente une position qui peut être défendue seulement si la valeur de la justice est vue comme étant supérieure à toutes les autres valeurs. Ceux qui sont d'accord avec cet argument croient que la sécession est réclamée dans le but d'instaurer la justice. Les auteurs qui défendent le droit à la sécession voient plutôt la justice comme étant dépendante de l'auto-détermination et c'est pourquoi ils ne sont pas d'accord avec cet argument rawlsien. Celui-ci est remis en question par le deuxième argument de la section des arguments favorables.

Troisièmement, un risque d'inclure le droit à la sécession dans la Constitution est que cela pourrait faire en sorte qu'il y ait davantage de conflits ethniques (Sunstein, 1991, p. 634). En effet, il est possible d'imaginer que cela diviserait les entités fédérées et mènerait à de potentielles frictions. Il m’apparaît pourtant évident qu'un conflit ethnique imminent surviendra peu importe si un droit à la sécession est inscrit dans la Constitution. Par ailleurs, s'il se trouve que c'est bel et bien le fait de détenir un droit à la sécession qui fait en sorte qu'une entité fédérée fasse sécession, il est légitime pour une entité fédérée de réclamer son droit à l'auto-détermination et de l'exercer. Ce droit est vu plus précisément dans le deuxième argument de la section des arguments favorables.

\section{Arguments en faveur et point de vue personnel}

Les arguments contre présentés ci-dessus sont pertinents pour mieux comprendre les arguments en faveur puisque l'un constitue souvent une réplique à l'autre. J'ai répertorié cinq arguments favorables à l'inclusion d'un droit à la sécession dans la

Constitution d'un État. L'idée première de laquelle découlent tous les autres arguments est 
l'avis que le " droit de chaque nation ou de chaque peuple de déterminer son propre destin " est plus qu'important, il est fondamental (Brilmayer, 1991, p. 179).

Premièrement, un droit à la sécession devrait être inclus dans la Constitution, afin de servir comme " contrepoids au pouvoir coercitif et monopolistique de l'État» (Kreptul, 2003, p. 55). En effet, l'État central devrait être plus sensible aux revendications des entités fédérées qui le constituent puisque, en tout temps, l'État central devrait garder en tête qu'une entité fédérée pourrait recourir au droit à la sécession. Cela pourrait se produire si le gouvernement central ne respecte pas certaines ententes prises avec les États fédérés, je pense par exemple à des promesses électorales. Cet argument rejoint directement le premier argument contre l'inclusion d'un droit à la sécession présenté dans ce travail. En effet, celui-ci veut que le droit à la sécession soit vu comme une nuisance dans les débats politiques, tandis que l'argument pour considère plutôt ce droit comme un avantage pour les États fédérés. Les conséquences imaginées ne sont donc pas du tout les mêmes. Dans l'idée que le droit à la sécession agirait comme contrepoids, il semble clair que ce droit est un avantage puisqu'il permettrait aux entités fédérées de faire valoir davantage leurs intérêts. Je suis en accord avec cette vision du fédéralisme, car, comme défini dans la première partie de ce texte, il doit garantir que « chaque État puisse conserver son intégrité politique fondamentale» (Encyclopaedia Britannica, 2016). Selon cette définition, l'État central n’a donc pas la capacité d'obliger une entité fédérée à rester sous sa gouverne. Je suis d'accord avec cet argument, car, comme le présente Sunstein dans son contreargumentaire, « il n’y a pas de bonnes raisons pour croire que [les effets négatifs] vont se produire " (Sunstein, 1991, p. 653). Dans une fédération qui a été créée de façon juste afin que chaque entité fédérée bénéficie de l'union fédérale, les entités fédérées ne réclameront le droit à la sécession que dans des cas extrêmes. En prenant le cas du Canada où le droit à la sécession s'est précisé avec le Renvoi relatif à la sécession du Québec, il serait faux de dire que les revendications sécessionnistes ont augmenté depuis la publication de l'avis de la Cour suprême. Par ailleurs, même si plusieurs entités fédérées désiraient se retirer d'une union fédérale, il faudrait respecter les gouvernés et c'est ce qui m'amène au deuxième argument favorable à l'inclusion du droit à la sécession dans la Constitution.

Pour le deuxième argument, j’abonde dans le même sens que Ludwig von Mises qui soutient que ce qui doit primer est « le consentement des gouvernés» (Kreptul, 2003, p. 57) 
plutôt que de voir la justice comme une conséquence logique d'un "État démocratique constitutionnel ", comme le veut le deuxième argument contre (Rawls cité par Kreptul, 2003, p. 45). Altman et Wellman croient que même dans les «État parfaitement légitimes", "les nations peuvent avoir un droit primaire à la sécession " (Altman et Wellman, 2009, p. 5). C'est plutôt l'acceptation du contrat social accepté par les gouvernés qui prime. Par conséquent, si une population vote en faveur d'une sécession, celle-ci doit avoir lieu (Kreptul, 2003, p. 57). L'idée de philosophie politique du contrat social veut que la gouverne du peuple soit acceptée parce que la population y a consenti implicitement. S'il arrive qu'une opposition explicite d'une majorité d'une population soit exprimée par rapport à un gouvernement et qu'un désir de sécession est clairement établi, il serait contre l'idée même du contrat social que d'obliger un groupe de personnes à vivre dans un État qu'ils ont refusé. Pour expliquer la pertinence de cet argument, il importe de revenir à la base de l’idée fédérale. Bednar et al. évoquent que plusieurs fédérations ont cessé de fonctionner lorsque des entités fédérées " ont décidé que les bénéfices de faire partie de la fédération ne valaient plus la peine, en termes de santé économique, de sécurité ou d'autonomie régionale» (Bednar et al., 2001, p. 3). Lorsque les inconvénients excédent les bénéfices, il devient fort probable que les gouvernés réalisent qu'ils seraient mieux sans la fédération, et il faut alors respecter leur choix de se retirer, de la même façon que la fédération ne les a pas obligé à s'y joindre à sa création.

Le troisième argument en faveur de l'inclusion du droit à la sécession dans la Constitution est le suivant : il serait avantageux pour tous les partis de savoir à l'avance les modalités dans le cas hypothétique d'un désir de sécession. En effet, prévoir le procédé à l'avance permettrait d'engendrer une "transformation légale et pacifique " si l'envie de sécession se concrétise (Kreptul, 2003, p. 87). Le cas du Québec et du Canada illustre parfaitement la nécessité de prévoir le droit à la sécession et ses modalités. Ce cas complexe concernant une potentielle sécession du Québec du Canada comporte plusieurs traits distinctifs. La Cour suprême du Canada a rendu un jugement qui parle d'une "majorité claire ». Comme on pouvait l'imaginer, les politiciens des deux principaux camps de cet enjeu québécois ne s'entendent toujours pas sur ce que signifient ces deux mots (Morissette, 2001, p. 80-93). Si, avant que ne surgissent les revendications pour la sécession du Québec, les différentes entités politiques s'étaient entendues sur le procédé pour appliquer le droit à 
la sécession, l'avis demandé à la Cour suprême n'aurait pas eu lieu d'être. En effet, d'un point de vue moral, mais aussi légal, si les modalités ont été inscrites clairement, l'État central et les entités fédérées deviennent alors obligés de respecter l'entente préétablie même si cela ne leur convient pas. Daniel Weinstock soutient cet argument et transpose le concept du voile de l'ignorance de Rawls à l'incorporation du droit à la sécession dans une Constitution (Kreptul, 2003, p. 54). Weinstock explique que le choix des modalités du droit à la sécession se ferait par « les participants [...] sans savoir s'ils font partie d'un groupe national minoritaire ou majoritaire" (Weinstock paraphrasé par Kreptul, 2003, p. 54). Reproduire le voile de l'ignorance afin de décider des modalités constitutionnelles d'un droit à la sécession permettrait, explique-t-il, de trouver une position rationnelle et « équilibrée " (Kreptul, 2003, p. 54). Hoffman se questionne à ce sujet et écrit, à propos du voile de l'ignorance : « La société internationale se compose à la fois d'individus [...] et d'États. [...] Les délégués placés sous le voile d'ignorance représentent-ils les États ou les individus de la planète? " (Hoffman, 1996, p. 104-105). À mon avis, cette question importe peu dans le cas présent. Que ce soit des individus ou des États qui décident des modalités du droit à la sécession, une position raisonnable devrait être atteinte parce que les décideurs ne connaitront pas leur position dans la fédération. Il me semble grandement difficile d'imaginer que quelqu'un soit contre cet argument, puisqu'il relève de la bonne foi et, en utilisant le voile de l'ignorance, ne favorise aucun parti. Reconnaitre l'importance de l'inclusion du droit à la sécession et de ses modalités dans la Constitution est une façon de participer à la joute politique dans les règles de l'art, c'est-à-dire en n'essayant pas de prendre le dessus en omettant un droit fondamental dans le texte de loi suprême de l'État.

Le quatrième argument en faveur de l'inclusion d'un droit à la sécession inscrit dans la Constitution des fédérations est que ce droit découragerait l'utilisation de "pratiques oppressives et discriminatoires" envers des groupes ethniques ou des entités fédérées (Sunstein, 1991, p. 635). En effet, ce droit donnerait un poids supplémentaire aux entités fédérées. Celles-ci pourraient invoquer ce droit lorsqu'elles sentent que les mesures prévues par l'État central leur nuisent et qu'elles auraient avantage à faire sécession. Par exemple, l'État central ne pourrait pas se permettre de prendre constamment des décisions désavantageant certaines entités fédérées simplement parce que ces entités fédérées sont des minorités ou sont les boucs-émissaires des autres habitants de la fédération. Par 
ailleurs, si des positions prises par le gouvernement central opprimaient certaines entités fédérées, ces dernières auraient un plus grand poids en disant qu'elles peuvent décider de quitter l'union si celle-ci n'est pas assez profitable. L'équilibre parmi les entités fédérées reste toutefois présent, puisque chaque entité possède le même droit à la sécession. Je suis d'avis que les entités fédérées doivent avoir ce droit, car, dans ma vision du fédéralisme, les entités fédérées doivent sentir qu'elles retirent un bénéfice de l'union fédérale et non pas qu'elles sont considérées comme des minorités ou qu'elles sont exclues (Bednar et al., 2001, p. 3). Bednar et al. expliquent que «tous doivent croire que les gouvernements régionaux n'essaieront pas de prendre avantage sur l'un ou l'autre et que le gouvernement central n'essaiera pas d'usurper le pouvoir des régions ", puisque ceci serait une raison valable de revendiquer la sécession (Bednar, Eskridge et Ferejohn, 2001, p. 2). Les entités fédérées ne doivent pas se sentir en compétition avec les autres entités fédérées.

Finalement, le cinquième et dernier argument en faveur de l'inclusion d'un droit à la sécession dans les Constitutions des fédérations est que "certains droits sont enchâssés parce que les individus devraient être autorisés à les exercer indépendamment de ce que les majorités peuvent penser" (Sunstein, 1991, p. 637). Ce droit est vu comme étant "complètement indépendant de la démocratie» et comme étant un droit qui devrait toujours être en place et que les idées à la mode ne devraient pas influencer (Sunstein, 1991, p. 637). Le droit à la sécession devrait être inclus dans la Constitution de toutes les fédérations puisqu'il s'agit d'un droit qui doit prévaloir en tout temps et qui ne doit pas être écarté selon une crise politique ponctuelle. Cela rejoint le troisième argument favorable concernant l'importance d'inclure les modalités afin de savoir à quoi s'en tenir dans le cas de revendications sécessionnistes. Inscrire ce droit dans la Constitution, c'est reconnaître qu'il fait partie d'une classe de droits à part qui n'ont pas à être garantis par un politicien ou même par une loi ordinaire, mais qui sont garantis en tout temps par le texte de loi suprême d'un pays qu'est la Constitution (Sunstein, 1991, p. 637). Ce droit concerne le futur d'un peuple et son auto-détermination est c'est pour cette raison qu'il est aussi important. Le désir d'établir un droit à la sécession dans la Constitution d'un État est le symbole de l'importance donnée à l' " expression d'une autonomie collective » (Miller, 1995, p. 9). Miller précise que le regroupement créé dans le but de «façonner le monde" se fait " avec ceux avec lesquels ils s'identifient» (1995, p. 9). Suivant cette logique, si la population d'une 
entité fédérée souhaite s'auto-déterminer en recourant au droit à la sécession, il devrait être permis de le faire, puisque c'est son droit d'être représenté par la communauté politique qu'elle-même choisit. Si une nation tient à la sécession dans le but d'avoir une voix qui lui corresponde à l'international, cette " autonomie collective » doit être respectée et écoutée.

\section{Conclusion}

Dans l'avis que je présente qui est en faveur de l'inclusion d'un droit à la sécession, les définitions et les justifications des différents termes, mais surtout du fédéralisme, sont primordiales. Les arguments présentés qui s'opposent à la présence du droit à la sécession dans la Constitution des fédérations sont pertinents, mais entrent en contradiction avec les arguments favorables. En effet, certains des arguments sont opposés parce qu'ils découlent de valeurs différentes. Les valeurs que je considère comme essentielles se reflètent dans ma prise de position, à savoir l'importance de l'auto-détermination des peuples, l'honnêteté politique ainsi que la légitimité des revendications.

Les cinq arguments que je défends sont directement en lien avec ces valeurs. D'abord, je défends l'inclusion d'un droit à la sécession dans les Constitutions des fédérations, afin de créer un contrepoids supplémentaire au pouvoir de l'État central (Kreptul, 2003, p. 55). Cette idée permet d'ajouter du poids aux revendications des entités fédérées. Ensuite, je défends la primauté du « consentement des gouvernés » (Kreptul, 2003, p. 57). À mon sens, les gouvernés constituent les habitants de la province, du canton, ou de l'État qui doivent avoir leur mot à dire lors d'une possible sécession. Le choix du gouvernement revient aux habitants et ce choix doit pouvoir être changé en tout temps. Le troisième argument que je présente est l'importance de connaître les règles du jeu. Avant de s'unir pour créer une fédération, l'État central et les entités fédérées doivent savoir ce qu'il adviendra en cas de revendications sécessionnistes sérieuses (Kreptul, 2003, p. 87). C'est ici que j'aborde le principe du voile de l'ignorance transposé à cet enjeu. Ensuite, l'argument suivant concerne la discrimination envers certaines entités fédérées (Sunstein, 1991, p. 635). Puisque les entités fédérées auraient une menace potentielle à sortir en cas de discrimination, soit le droit à la sécession, l'État central devrait être plus respectueux de leurs intérêts et de leurs requêtes. Le dernier argument que je présente est que le droit à la 
sécession est un droit si important qu'il devrait être inscrit dans la Constitution afin de rendre plus difficile de le retirer (Sunstein, 1991, p. 637).

Avant de terminer cet article, j'aimerais faire part d'une approche favorable au droit à la sécession, mais pas à son inclusion dans la Constitution. Au cours de l'écriture de cet argumentaire, je me suis questionnée et j'ai pensé qu'il s'agissait peut-être d'une erreur que de souhaiter voir ce droit inscrit dans les Constitutions. La distinction est que je ne revendique non seulement l'inclusion du droit dans la Constitution, mais aussi la présence de modalités l'entourant. La raison de mon doute est simple : les modalités n'avantageront peut-être pas les entités qui souhaitent se séparer du reste du pays. En effet, en suivant le concept du voile de l'ignorance appliqué au droit à la sécession, présenté par Weinstock, peut-être que le choix final demanderait, par exemple, un référendum dans toute la fédération et que l'option favorable à la sécession doive remporter plus de $75 \%$ des voix lors d'un référendum. Certains penseront que je ne respecte pas les règles du jeu en parlant de règles qui avantagent et désavantagent les entités fédérées. Pourtant, cette réalité existe et n’a pas pu être mieux mise en évidence que dans les débats qui ont suivi le Renvoi relatif à la sécession du Québec. À la suite de ma réflexion, je maintiens toutefois ma position; je pense qu'il serait tout de même cohérent d'accepter le choix résultant de ce principe rawlsien, et ce, principalement pour deux raisons. D'abord, ce n'est pas parce que les modalités pour accéder à la sécession sont plus difficiles à atteindre qu'il est impossible de les atteindre. Ensuite, tel que mentionné précédemment, je trouve essentiel d'avoir une honnêteté politique et d'être de bonne foi, il serait donc contraire à ce principe que de ne pas accepter le résultat du voile de l'ignorance si ce principe a été accepté en toute connaissance de cause. En bref, malgré cette hésitation, je reste fidèle à l'idée que j'ai défendue au cours de cette recherche, et je continue à supporter l'inclusion d'un droit à la sécession dans les Constitutions des fédérations. 


\section{April 14, 2017 FEDERALISM-E}

\section{Bibliographie}

ALTMAN, Andrew \& Christopher WELLMAN. 'Secession', dans A Liberal Theory of International Justice, Oxford, Oxford University Press, 2009, p. 43-68.

BERNAR, Jenna, William N. ESKRIDGE Jr. et John FEREJOHN. 'A Political Theory of Federalism', dans Constitutional Culture and Democratic Rule. New York, Cambridge University Press, 2001, p. 223-270.

BRILMAYER, Lea. 'Secession and Self-Determination: a Territorial Interpretation', Yale Journal of International Law, Vol. 16, 1991, p. 177-202.

Encyclopaedia Britannica. Federalism, [En ligne], (2016), https://www.britannica.com/topic/federalism, $1^{\mathrm{er}}$ novembre 2016

DAHHLITZ, Julie. Secession and International Law: Conflict Avoidance - Regional Appraisals. New York, United Nations Publications, 2003, 283 p.

ÉTHIER, Diane. Introduction aux relations internationales. Montréal, Les Presses de l'Université de Montréal, 2004, 298 p.

HOFFMANN, Stanley. 'Mondes idéaux', dans John RAWLS. Le droit des gens. Paris, Éditions Esprit, 1996, p. $97-$ 129.

KREPTUL, Andrei. 'The Constitutional Right of Secession in Political Theory and History', Journal of Libertarian Studies, Vol. 17(4), 2003, p. 39-100.

MILLER, David. 'National Self-Determination', dans On Nationality. Oxford, Oxford University Press, 1995, p. 81-118.

MORISSETTE, Yves-Marie. Le Renvoi sur la sécession du Québec. Montréal, VARIA, 2001

SUNSTEIN, Cass R. 'Constitutionalism and Secession', The University of Chicago Law Review, Vol. 58(2), 1991, p. 633-670. 